\title{
An improved torque type gravity gradiometer with dynamic modulation
}

\author{
Jie Luo ${ }^{1} \cdot$ Jia-Hao $\mathrm{Xu}^{1} \cdot \mathrm{Qi} \mathrm{Liu}^{2} \cdot$ Cheng-Gang Shao ${ }^{3} \cdot$ \\ Lin $\mathrm{Zhu}^{3} \cdot$ Hui-Hui Zhao ${ }^{3}$. Wei-Huang $\mathrm{Wu}^{1}$
}

Received: 29 December 2016/Accepted: 7 July 2017/Published online: 24 July 2017

(C) Akadémiai Kiadó 2017

\begin{abstract}
Traditional torque type gravity gradiometer has an important pole in gravity gradient measurements, while it is relatively inefficient and with the precision of about $1 \mathrm{E}$ mainly caused by the static operating mode. In this paper, we develop an improved torque type gravity gradiometer to improve the measuring efficiency, which is based on the dynamic modulation. The dynamic modulation keeps the gradiometer rotating on a turntable steadily, measures the deflection angle of the torsion pendulum continuously and then obtains the gravity gradients. The result shows that after using the improved gradiometer, the gradients $W_{x z}$ and $W_{y z}$ are obtained with precisions of $0.45 \mathrm{E}$ and $0.32 \mathrm{E}$ respectively in a cycle of $20 \mathrm{~min}$.
\end{abstract}

Keywords Improved torque type gradiometer - Dynamic modulation · Measuring efficiency · Gravity gradient

\section{Introduction}

The gravity gradient measurement can offer an efficient way for resource explorations in earth (Zhou et al. 2015; Völgyesi 2001). The torque type gradiometer was widely applied to the oil field exploration in the past (Bell and Hansen 1998; Szabó 2016), because the

Qi Liu

louis_liuqi@mail.hust.edu.cn

$\triangle$ Cheng-Gang Shao

cgshao@mail.hust.edu.cn

1 School of Mechanical Engineering and Electronic Information, China University of Geosciences, Wuhan 430074, China

2 TIANQIN Research Center for Gravitational Physics, School of Physics and Astronomy, Sun Yatsen University, Zhuhai 519000, China

3 MOE Key Laboratory of Fundamental Physical Quantities Measurement, School of Physics, Huazhong University of Science and Technology, Wuhan 430074, China 
gravity gradient data could be used to reveal underground mass distribution. The torque type gradiometer designed by Eötvös made a great difference on the petroleum industry in early twentieth century (Barton 1931; DiFrancesco et al. 2009; Shaw and Lancaster-Jones 1922). The equilibrium position and torsional motion of the pendulum in this type gradiometer were found to be remarkably stable and relatively constant, so that the instrument could be used not only in a well-protected laboratory, but also in a field (Shaw and Lancaster-Jones 1922). However, the gravity gradient measurement with the Eötvös torque type gradiometer needs a long time to record the deflection angle of the torsion pendulum. The observation using this type gradiometer should be done in the night of stable measurement environment (Szabó 2016), since it is easily affected by the external environment variations, such as temperature fluctuation, barometric pressure change and so on. There have been many successive improvements on the instrument structure and measuring process, while all these relative observations with the static operating mode need a long measurement cycle (Schweydar 1918; Rankine 1932). The gravity gradients given by the torque type gradiometer should be determined by observing at least five different azimuth angles (Bell and Hansen 1998), the observers must alternate the azimuth angles of the gradiometer by a turntable with manual and later automatic operations (Völgyesi 2015), and besides the formal observation starts after the torsion pendulum reach to the stable state. Typically, the torsion pendulum has a period of swing exceeding twenty minutes (Rankine 1932). These above factors result in the relatively low efficiency of this type gradiometer. Then, for this traditional torque type gradiometer, the gravity gradients could be obtained with a precision of $1 \mathrm{E}$ after $12 \mathrm{~h}$ observation (Shaw and LancasterJones 1922).

In order to improve the measurement efficiency and accuracy, we develop an improved torque type gradiometer with dynamic modulation mode to determine the gravity gradients. The torsion pendulum is placed on a stable turntable, which rotates continuously at a constant velocity during the observation, and then we can obtain a set of gravity gradients from every rotating cycle. In addition, the torsion pendulum is free to twist, and the useful signal is modulated on the measurable deflection angle (Luo et al. 2013). Therefore, compared to the traditional Eötvös torque type gradiometer with the static operating mode, the torsion pendulum of the improved gradiometer can observe the deflection angle of the pendulum continuously and measure gravity gradients in a short period of the turntable. Due to the short measurement cycle, this improved Eötvös torque type gradiometer can avoid unnecessary noises and disturbances. In this paper, we propose the principle of the improved gradiometer, describe the design of the relative instrument, and analyze the influences of the thermal noise and irregularity in the rotation rate on the estimation of the gravity gradients. Finally, we process a typical data set of the measurement of the gravity gradients with the improved torque type gradiometer, then obtain the values and uncertainties of the gravity gradients, and further make contribution to the determination of the gravity gradients.

\section{Principle of the improved torque type gradiometer with dynamic modulation}

\subsection{Gravity gradient}

The gravity potential $W$ of the earth is expressed as 


$$
W=V+U,
$$

where $V$ is the gravitational potential, $U$ is the potential of centrifugal force. There are $9 \mathrm{~s}$ order partial derivatives of $W$ in a cartesian coordinate system, expressed as:

$$
\nabla^{2} W=\left(\begin{array}{l}
\frac{\partial^{2} W}{\partial x^{2}} \frac{\partial^{2} W}{\partial x \partial y} \frac{\partial^{2} W}{\partial x \partial z} \\
\frac{\partial^{2} W}{\partial y \partial x} \frac{\partial^{2} W}{\partial y^{2}} \frac{\partial^{2} W}{\partial y \partial z} \\
\frac{\partial^{2} W}{\partial z \partial x} \frac{\partial^{2} W}{\partial z \partial y} \frac{\partial^{2} W}{\partial z^{2}}
\end{array}\right)=\left(\begin{array}{c}
W_{x x} W_{x y} W_{x z} \\
W_{y z} W_{y y} W_{y z} \\
W_{z x} W_{z y} W_{z z}
\end{array}\right)
$$

where the five components $W_{x x}, W_{x y}, W_{x z}, W_{y y}$, and $W_{y z}$ are independent, since the earth gravity field is an irrotational field and the three diagonal components satisfy the Poisson equation in the earth (Dehlinger 1978; Völgyesi 2015). The gravity gradients are defined as $W_{i j}(i, j=x, y, z)$, which contain more detailed information of the gravity potential than first order partial derivatives of $W$.

\subsection{Principle of the improved gradiometer with dynamic modulation}

As the Fig. 1 shows, our specific torsion pendulum consists of four identical cylindrical test masses made of aluminum (diameter: $15.50 \mathrm{~mm}$, height: $13.42 \mathrm{~mm}$, mass: $2.53 \mathrm{~g}$ ), with essentially the same mass. They are positioned on a circular aluminum pendulum tray (diameter: $80 \mathrm{~mm}$, thickness: $2.5 \mathrm{~mm}$, mass: $33.93 \mathrm{~g}$ ). The masses 1 and 4 marked as the black circles in Fig. 1 are above the tray, the masses 2 and 3 marked as the black dashed circles are under the tray, and the four masses form a square with the side length $s$. The selection of arrangement for four test masses is beneficial for us to ensure the pendulum's sensitivity of the $W_{\mathrm{xz}}$ and $W_{\mathrm{yz}}$. We define a clear relation between the lab frame $(O-x y z)$ and the rotating frame $(O-X Y Z)$ in order to describe the principle of the dynamic modulation explicitly. Both origins $O$ of the above two frames are at the same center-of-mass of

Fig. 1 Schematic drawing of the pendulum tray, the four test masses and the autocollimator viewing from the top. The $s$ equals $42.23 \mathrm{~mm}$. Both origins $O$ of the above two frames are at the same center-of-mass of the torsion pendulum. The $x(X)$ and $y(Y)$ are axes of the lab (rotating) frame. The $X$ and $Y$ are along the direction of test masses $2 \rightarrow 1$ and $1 \rightarrow 4$ respectively. The $x$ and $y$ are towards East and North respectively

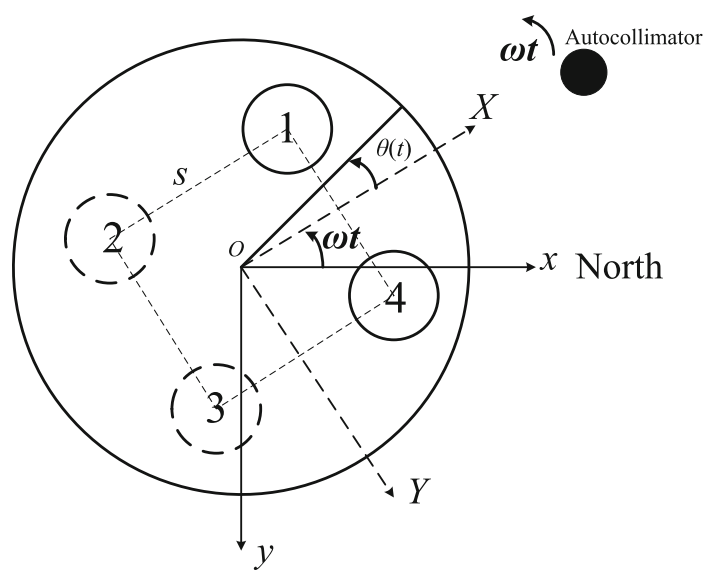

East 


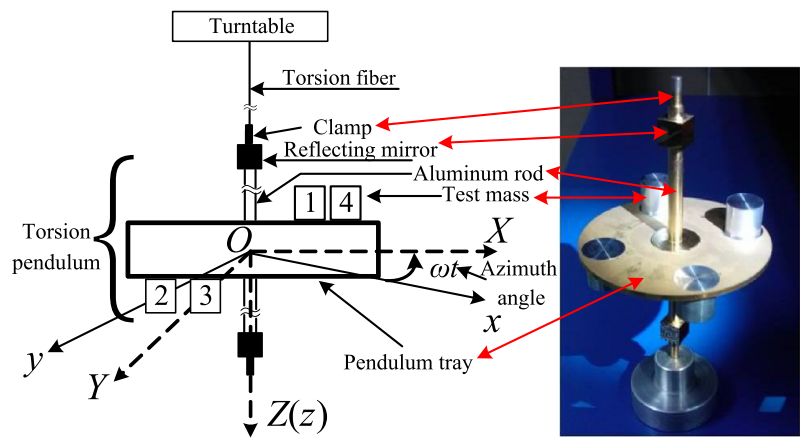

Fig. 2 (color online) Schematic drawing of the torsion pendulum, viewing from the front. The schematic drawing correspond to the photo of the pendulum. The torsion fiber and the two frames we defined are shown. The torsion pendulum consists of the two same clamps denoted as the black rectangles, two reflecting mirrors marked as the black squares, two same aluminum rods, four same test masses marked as the rectangle frame and a pendulum tray shown as the black rectangle frame. The black full line is the torsion fiber. The two frames are marked as the black solid and dashed lines with arrows, respectively. The azimuth angle between the two frames is marked as the $\omega t$

the torsion pendulum which rotates with the turntable together. The $X$-axis and $Y$-axis of the rotating frame $(O-X Y Z)$ are always along the directions of mass 2 pointing to mass 1 and mass 1 pointing to mass 4 , respectively. The vertical axes $z$ and $Z$ coincide with the direction along the torsion fiber marked as the black line in Fig. 2, parallel to the net force on the pendulum. The horizontal axes of the lab frame, $x$ and $y$, are parallel to North and East respectively in geodesy. As the Fig. 2 shows, in our dynamic modulation measurement, we adjust the two frames to coincide with each other when the pendulum is in the equilibrium position, which occurs before we start to measure the gravity gradients. Then, we keep the pendulum rotating with the turntable at a constant angular velocity $\omega$. The $t$ is the dynamic modulation time, and the azimuth angle $\omega t$ is the rotation angle of the rotating frame relative to the fixed lab frame in the dynamic modulation. The deflection angle $\theta(t)$ of the pendulum is measured in rotating frame by an autocollimator which rotates together with the pendulum counterclockwise.

The inertia tensors of the pendulum, $I_{L M}(L, M=X, Y, Z)$ can be calculated by the physical parameter of the pendulum in the $O-X Y Z$, and they are constant because both of the pendulum and the rotating frame rotate together with the turntable. In other words, the relative position between pendulum and the rotating frame is constant.

The torque about the fiber $\tau(t)$ caused by the gravity gradients on the whole pendulum is (Völgyesi 2015)

$$
\tau(t)=\left(I_{Y Y}-I_{X X}\right) \cdot W_{X Y}+I_{X Z} \cdot W_{Y Z},
$$

where $W_{X Y}, W_{X Z}$ are gravity gradient components defined in the $O-X Y Z$. The $W_{X Y}$ and $W_{X Z}$ are just intermediate physical quantities and change in the different azimuth angle. These time-varying gravity gradient components should be converted into some gravity gradient components which are defined in fixed lab frame $O-x y z$. The relation between two frames is written as 


$$
\left\{\begin{array}{l}
X=x \cos (\omega t)+y \sin (\omega t) \\
Y=y \cos (\omega t)-x \sin (\omega t) \\
Z=z
\end{array} .\right.
$$

Then Eq. (3) can be rewritten as:

$$
\begin{aligned}
\tau(t)= & \left(I_{Y Y}-I_{X X}\right) \sin (2 \omega t) / 2 \cdot\left(W_{y y}-W_{x x}\right)+\left(I_{Y Y}-I_{X X}\right) \cos (2 \omega t) \cdot W_{x y} \\
& +I_{X Z}\left(\cos (\omega t) \cdot W_{y z}-\sin (\omega t) \cdot W_{x z}\right),
\end{aligned}
$$

where $W_{x x}, W_{y y}, W_{x y}, W_{x z}$ and $W_{y z}$ are gravity gradients defined in the $O-x y z$. The pendulum has a tiny deflection angle $\theta(t)$ under the torque $\tau(t)$, and the torque is expressed as:

$$
\tau(t)=k \theta(t),
$$

where $k$ is the torsional spring constant of the fiber (Tu et al. 2010).

In this way, we could observe the $\theta(t)$ to obtain the torque about the fiber, and then measure the gravity gradient components defined in the $O-x y z$. The observed deflection angle of the pendulum is expressed as:

$$
\begin{aligned}
\theta(t)= & \left(I_{Y Y}-I_{X X}\right)\left(W_{y y}-W_{x x}\right) \sin (2 \omega t) /(2 k)+\left(I_{Y Y}-I_{X X}\right) W_{x y} \cos (2 \omega t) / k \\
& +I_{X Z}\left(\cos (\omega t) W_{y z}-\sin (\omega t) W_{x z}\right) / k
\end{aligned}
$$

Equation (7) shows that the $\theta(t)$ is a sinusoidal function of the turntable rotation angle $\omega t$. And the $\theta(t)$ consisting of $1 \omega, 2 \omega$ orthogonal signals with four coefficients of the sine signal amplitude components $a_{1}^{\text {sin, }} \quad a_{2}^{\text {sin }}$ equals $-\left(I_{X Z} / k\right) \cdot W_{x z}$ and $\left(\left(I_{Y Y}-I_{X X}\right) /\right.$ $(2 k)) \cdot\left(W_{y y}-W_{x x}\right)$, respectively. The cosine signal amplitude components $a_{1}^{\cos }, a_{2}^{\text {cos }}$ equals $\left(I_{X Z} / k\right) \cdot W_{y z}$ and $\left(\left(I_{Y Y}-I_{X X}\right) / k\right) \cdot W_{x y}$, respectively. For every cycle of rotation, we can estimate $a_{1}^{\text {sin }}, a_{1}^{\text {cos }}, a_{2}^{\text {sin }}$ and $a_{2}^{\text {cos }}$ accurately via the nonlinear least-squaring fitting method. Then, the three gravity gradient components $W_{x y}, W_{y z}$ and $W_{x z}$, and the linear combination of the independent components $\left(W_{y y}-W_{x x}\right)$ are given by:

$$
\left\{\begin{array}{l}
W_{y y}-W_{x x}=2 k /\left(I_{Y Y}-I_{X X}\right) a_{2}^{\sin } \\
W_{x y}=k /\left(I_{Y Y}-I_{X X}\right) a_{2}^{\cos } \\
W_{y z}=\left(k / I_{X Z}\right) a_{1}^{\cos } \\
W_{x z}=\left(-k / I_{X Z}\right) a_{1}^{\sin }
\end{array} .\right.
$$

For the traditional torque type gradiometer with the static operating mode, the $\theta$ is not a consecutive sinusoidal signal but five discrete data. The discrete data obtained from the observation of the $\theta$ at five difference azimuth angles (Lancaster-Jones 1932). Typically, this gradiometer sets the torsion pendulum at $0^{\circ}, 72^{\circ}, 144^{\circ}, 216^{\circ}, 288^{\circ}$ azimuth angles and then observe $\theta$ for each azimuth angle (Shaw and Lancaster-Jones 1922; Völgyesi 2015). In this way, observers could calculate the four gravity gradient components $\left(W_{y y}-W_{x x}\right)$, $W_{x y}, W_{y z}$ and $W_{x z}$ by the five independent equations based on Eq. (7). In this operating process, observers alternate azimuth angles of the pendulum regularly, and then the pendulum starts to swing. The torsion pendulum has a period of swing exceeding $20 \mathrm{~min}$, and after having been disturbed returns to rest in its almost equilibrium position in approximately $2 \mathrm{~h}$ (Shaw and Lancaster-Jones 1922). Besides, the $\theta$ is recorded only after the torsion pendulum at a almost steady state. Therefore, most of the observe time is used to wait for the pendulum reaching to its equilibrium position, and it needs a complete observe cycle of at least $12 \mathrm{~h}$. 
For the improved gradiometer with dynamic modulation, as above mentioned, the $\theta(t)$ is modulated as a sinusoidal signal by the rotating turntable. The recorded $\theta(t)$ is a real reflection of the external torque coming from the gravity gradients, and the signal is sampled from the deflection angle of the pendulum at uniformly-spaced azimuth angles continuously during the experiment. That is to say, the improved gradiometer increase the sampling rate, and then decrease the observing time. It avoids costing much time as the traditional torque type gradiometer does. For every observed cycle, it could obtain a periodic signal which consists of $1 \omega$ and $2 \omega$ sine and cosine signals just as Eq. (7) shows. Therefore, the four gravity gradient components can also be extracted from these sampled sinusoidal signals. The most distinct feature of the dynamic modulation measurement is that it needs less measurement time than the static operating mode. In this way, we could minimize the external fluctuations and improve the measurement accuracy. Besides, we can select a suitable rotation rate to avoid $1 \omega$ or $2 \omega$ signals mixing with the free torsion oscillation signal of the pendulum.

\section{Description of the gradiometer}

The scheme of the experimental apparatus used to perform the measurements is shown in Fig. 3. The main body of the torsion pendulum is suspended by an annealed tungsten fiber (Goodfellow Cambridge Limited) with a length of $1000 \mathrm{~mm}$ and a diameter of $25 \mu \mathrm{m}$, which hangs from a clamp which connects with an aluminum rod (long: $110 \mathrm{~mm}$, diameter: $6 \mathrm{~mm}$, mass: $2.67 \mathrm{~g}$ ). And the aluminum rod is through the center of the pendulum tray, and two reflecting mirrors and two clamps are installed on its bottom and top symmetrically. The total mass of the torsion pendulum equals $73.35 \mathrm{~g}$ which is a half of the max load of the fiber, and hence the fiber is maintained safe working status during the experiment. The top of the tungsten fiber connects to the vacuum chamber marked as the thick black full line in Fig. 3, which is fixed on the turntable. The turntable is driven by a stepper motor and fixed on a supporting frame. An ion pump, which locates on the vacuum chamber and rotates with the turntable, is used to maintain a pressure of $\sim 10^{-5} \mathrm{~Pa}$ (SP400 ) in the chamber during the experiments. Then the air damping can be negligible. An autocollimator (ELCOMAT 3000), which also locates on the vacuum chamber and rotates

Fig. 3 A front view of the improved gradiometer in our measurement. The pendulum, ion pump, autocollimator and torsion pendulum rotate together at a constant angular velocity $\omega$

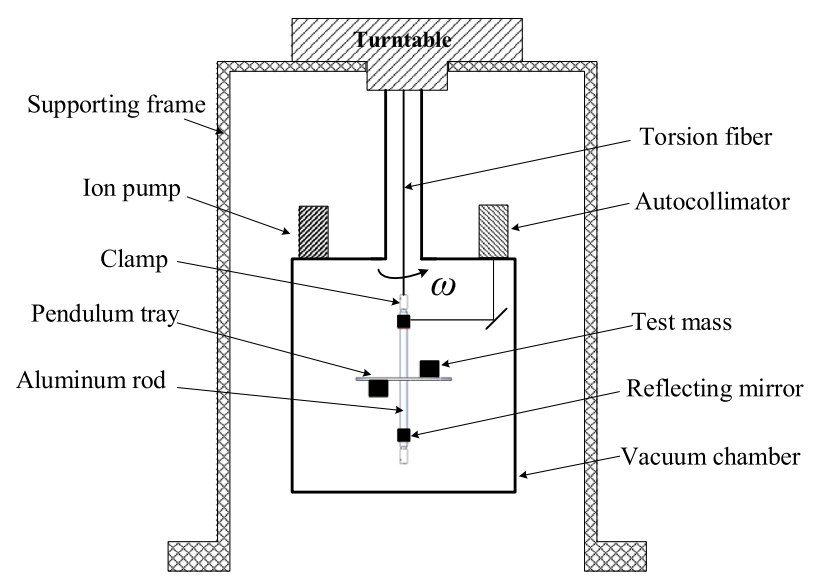


with the turntable, is used to measure the deflection angle $\theta(t)$ of the torsion pendulum. The inertia tensors of the pendulum are calculated in the $O-X Y Z$, and the $I_{X X}, I_{Y Y}, I_{X Z}$ and $I_{Z Z}$ are $45.404,45.404,4.620$ and $52.822 \mathrm{~kg} \mathrm{~mm}^{2}$ respectively. This installation leads to $I_{X X}=I_{Y Y}$, so we can measure $W_{x z}, W_{y z}$ based on Eq. (8) at present.

We divide the experiment into 4 steps. For the first step, we use the rotary vane pump, the turbo molecular pump and an ion pump to maintain a pressure of $10^{-5} \mathrm{~Pa}$ in the chamber during the experiment. For the second step, we let the two frames coincide when the pendulum is in the equilibrium position before formal measurement of the gravity gradients by adjusting the fiber. For the third step, the rotation period $T$ of the turntable drive motor is set $1200 \mathrm{~s}$, then the turntable drive motor is activated, and thus pendulum starts to rotate around the $Z$ axis slowly. Meanwhile, the rotating autocollimator records the discrete data of the deflection angle $\theta(t)$ at a regular interval of $1 \mathrm{~s}$. Finally, we obtain a angle-time data set $\left\{\theta\left(t_{i}\right), i=1,2, \ldots\right\}$, where $t_{i}$ is the sequence of sampling time.

\section{Systematic effects}

Thermal noise is one of the most fundamental limits to the precision of mechanical measurements, which is also of great importance and needs considering in the highsensitivity gradiometer. Besides, in the improved gradiometer, since we use the turntable which rotates continuously at a constant velocity during the observation, we should also consider the effect from the irregular rotation rate of the turntable on the gravity gradients.

\subsection{Thermal noise}

Thermal noise originates from Brownian motion, and the power spectrum of the $\theta(t)$ due to the thermal noise could be written as:

$$
|\theta(\omega)|^{2}=\frac{4 k_{B} \tilde{T} I_{Z Z} \omega_{0} Q}{\left(k-I_{Z Z} \omega^{2}\right)^{2} Q^{2}+I_{Z Z}^{2} \omega^{2} \omega_{0}^{2}},
$$

where $k_{B}, \tilde{T}$ and $Q$ are Boltzmann constant, ambient temperature and quality factor of the torsion balance system, respectively (Saulson 1990). And the resonant frequency $\omega_{0}$ equals $\sqrt{k / I_{Z Z}}$. In our experiment, the $Q$ of the fiber approximates to 3000, the $\tilde{T}$ approximates to $294 \mathrm{~K}$, the $k$ equals $6.2 \times 10^{-9} \mathrm{Nm} / \mathrm{rad}$ and the corresponding $\omega_{0}$ is about $0.0108 \mathrm{rad} / \mathrm{s}$. We plot the thermal noise power spectrum of the $\theta(t)$ in Fig. 4.

If the measuring frequency ranges from 0 to $0.001 \mathrm{~Hz}$, then thermal noise limit can be expressed as:

$$
\delta_{\text {potential }}^{T-\text { noise }} \theta=\sqrt{\int_{0}^{0.001 \mathrm{~Hz}}|\theta(f)|^{2} d f}=1.18 e^{-8} \mathrm{rad} .
$$

According to the principle of the error average distribution (Kirkup and Frenkel 2006)

$$
\delta^{T-n o i s e} a_{1}^{\text {sin }}=\delta^{T-\text { noise }} a_{1}^{\text {cos }}=\delta_{\text {potential }}^{T-\text { noise }} \theta / \sqrt{2},
$$

where $\delta^{T-\text { noise }} a_{1}^{\text {sin }}$ and $\delta^{T-\text { noise }} a_{1}^{\text {cos }}$ are the errors of the thermal noise on $a_{1}^{\text {sin }}$ and $a_{1}^{\text {cos }}$ respectively. From Eq. (8), the errors of the thermal noise to $W_{x z}$ and $W_{y x}$ is given as: 


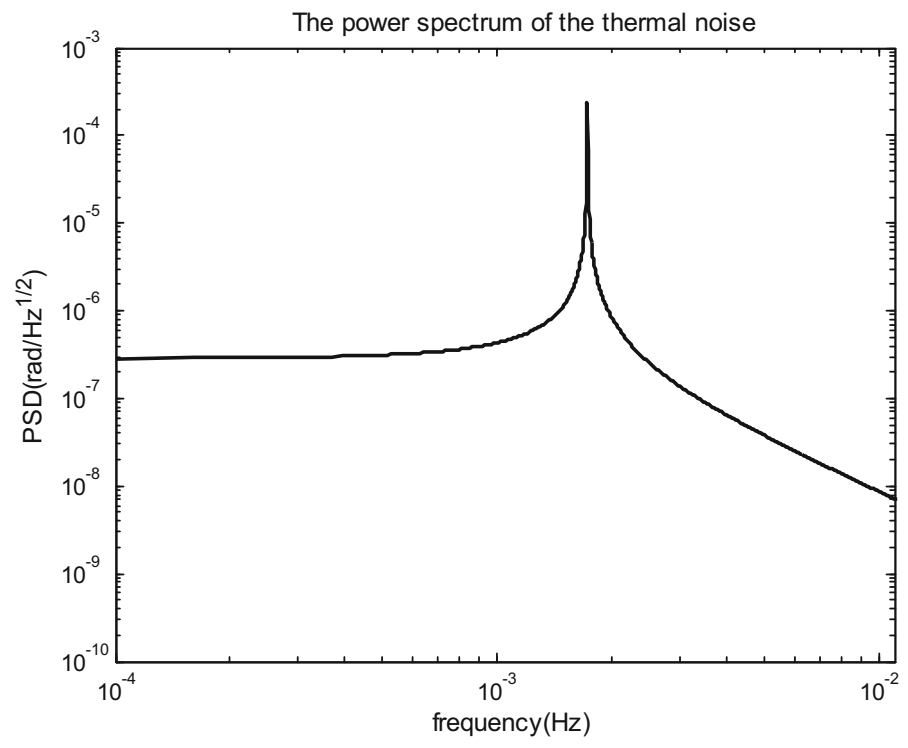

Fig. 4 The power spectrum of the thermal noise. The horizontal axis and vertical axis are the frequency and the amplitude of the power spectrum of the thermal noise

$$
\left\{\begin{array}{l}
\delta_{T-\text { noise }} W_{x z}=\frac{k}{I_{X Z}} \delta_{T-\text { noise }} a_{1}^{\text {sin }} \\
\delta_{T-\text { noise }} W_{y z}=\frac{k}{I_{X Z}} \delta_{T-\text { noise }} a_{1}^{\cos }
\end{array} .\right.
$$

As a result, our gradiometer measurement resolution for $W_{x z}$ and $W_{y x}$ are both $0.01 \mathrm{E}$ based on Eqs. (10-12).

\subsection{Irregularities in the rotation rate}

Suppose that the rotation of the turntable is not uniform, but it can be modulated at the rotation frequency $\omega$ and/or its higher harmonics $n \omega$. Then the actual turntable rotation angle $\alpha$ is expressed as:

$$
\alpha=\omega t+\sum_{n=1}^{\infty} \Phi_{n} e^{i n \omega t},
$$

where $\Phi_{n}$ are complex numbers. The deflection angle of the pendulum in the lab frame is defined as $\theta_{L}(t)$ (Su 1992; Choi 2006). Here the external torque is zero and the damping is ignored, and then the equation of the pendulum motion can be further written as ( $\mathrm{Su} \mathrm{1992):}$

$$
I_{Z Z} \ddot{\theta}_{L}=-k\left(\theta_{L}-\alpha\right)=-k\left(\theta_{L}-\omega t-\sum_{n=1}^{\infty} \Phi_{n} e^{i n \omega t}\right) .
$$


The solution of Eq. (13) is

$$
\theta_{L}=\omega t+\sum_{n=1}^{\infty} \frac{\omega_{0}^{2}}{\omega_{0}^{2}-n^{2} \omega^{2}} \Phi_{n} e^{i n \omega t}
$$

Therefore, in the rotating frame, the deflection angle $\theta$ is converted into:

$$
\theta=\theta_{L}-\alpha=\sum_{n=1}^{\infty} \frac{n^{2} \omega^{2}}{\omega_{0}^{2}-n^{2} \omega^{2}} \Phi_{n} e^{i n \omega t}
$$

If $n$ equals 1 , then there is a spurious $1 \omega$ signal due to the irregularities in the rotation rate. The coefficients of the sin and cosine components are given by

$$
\delta_{\text {turntable }} a_{1}^{\mathrm{sin}}=\frac{\omega^{2}}{\omega_{0}^{2}-\omega^{2}} \Phi_{1}, \quad \delta_{\text {turntable }} a_{1}^{\mathrm{cos}}=\frac{\omega^{2}}{\omega_{0}^{2}-\omega^{2}} \Phi_{1}
$$

The deviations $\delta_{\text {turntable }} W_{x z}$ and $\delta_{\text {turntable }} W_{y z}$ of the $W_{x z}$ and $W_{y z}$ due to irregularities in the rotation rate effect are expressed as:

$$
\left\{\begin{array}{l}
\delta_{\text {turntable }} W_{x z}=\frac{k}{I_{X Z}} \delta_{\text {turntable }} a_{1}^{\text {sin }} \\
\delta_{\text {turntable }} W_{y z}=\frac{k}{I_{X Z}} \delta_{\text {turntable }} a_{1}^{\mathrm{cos}}
\end{array},\right.
$$

It is necessary that the rotation rate of the turntable should be controlled at a single rotation frequency $\omega$. Typically, all of the complex numbers $\left|\Phi_{n}\right|$ are less $0.1 \mu \mathrm{rad}$, and then the deviations of the $W_{x z}$ and $W_{y x}$ due to irregularities in the rotation rate are $0.03 \mathrm{E}$ based on Eqs. $(15,16)$.

\section{Experiment result}

Before the formal measurement, we consider the measuring range of the improved gradiometer (Völgyesi and Ultmann 2012), but we do not find a point where the gravity gradients are big value in our lab. Since the range depends crucially on the measuring range of the autocollimator (ELCOMAT 3000), the range, namely the maximum measurable angle of the $\theta(t)$, of the ELCOMAT 3000 we adopted is $\pm 14,059.6 \mu \mathrm{rad}\left( \pm 2900^{\prime \prime}\right)$. According to the Eq. (8) e.g. $\sqrt{\left(a_{1}^{\cos }\right)^{2}+\left(a_{1}^{\sin }\right)^{2}} \leq 14059.6 \mu \mathrm{rad}$, the measuring range of the gradiometer is limited by an in equation $\sqrt{W_{x z}^{2}+W_{y z}^{2}} \leq 18873.4 \mathrm{E}$. It concludes that the gradiometer can measure $W_{\mathrm{xz}}$ and $W_{\mathrm{yz}}$ of a point where both of $\left|W_{\mathrm{xz}}\right|$ and $\left|W_{\mathrm{yz}}\right|$ are smaller than $13300 \mathrm{E}$.

After about forty-four hours formal measurement with the improved gradiometer, we obtain the angular deflection $\theta(t)$ of the torsion pendulum shown in Fig. 5. We find that $\theta(t)$ is not a simple sine (or cosine) signal, and it contains other frequency signals. Then, we plot the spectrum of the whole raw data shown in Fig. 5. We find that there are $1 \omega, 2 \omega$ and $\omega_{0}$ signals in the raw data. The $1 \omega$ signal is the useful oscillation signal for us to obtain $W_{x z}$ and $W_{y z}$. The $2 \omega$ signal is the second harmonic of the useful oscillation signal, caused by the defective of the pendulum(the inertia tensors $I_{X X}$ and $I_{Y Y}$ are not equal strictly) and the 

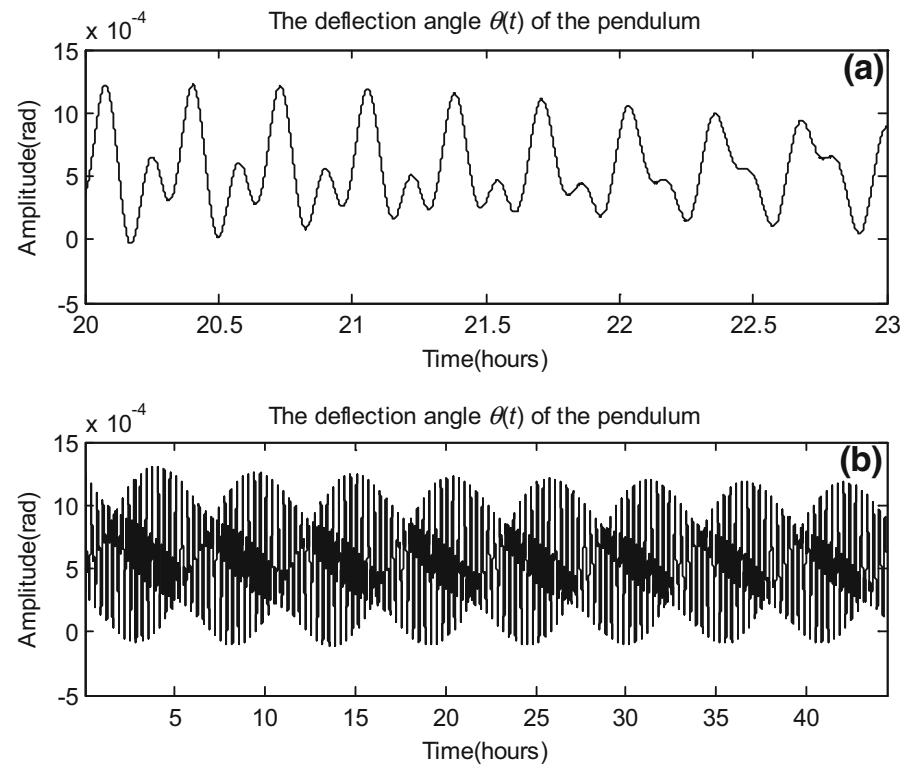

Fig. 5 The raw data $\theta(t)$ from $44 \mathrm{~h}$ (about 130 turntable periods) of a normal experiment. The black line is the signal $\theta(t)$. a The angular deflection $\theta(t)$ of the pendulum versus time at the time from 20 to $23 \mathrm{~h} ; \mathbf{b}$ The angular deflection $\theta(t)$ of the pendulum versus time during the whole measurement. The horizontal axis is the sampled time series, and the vertical axis is the amplitude of the $\theta(t)$

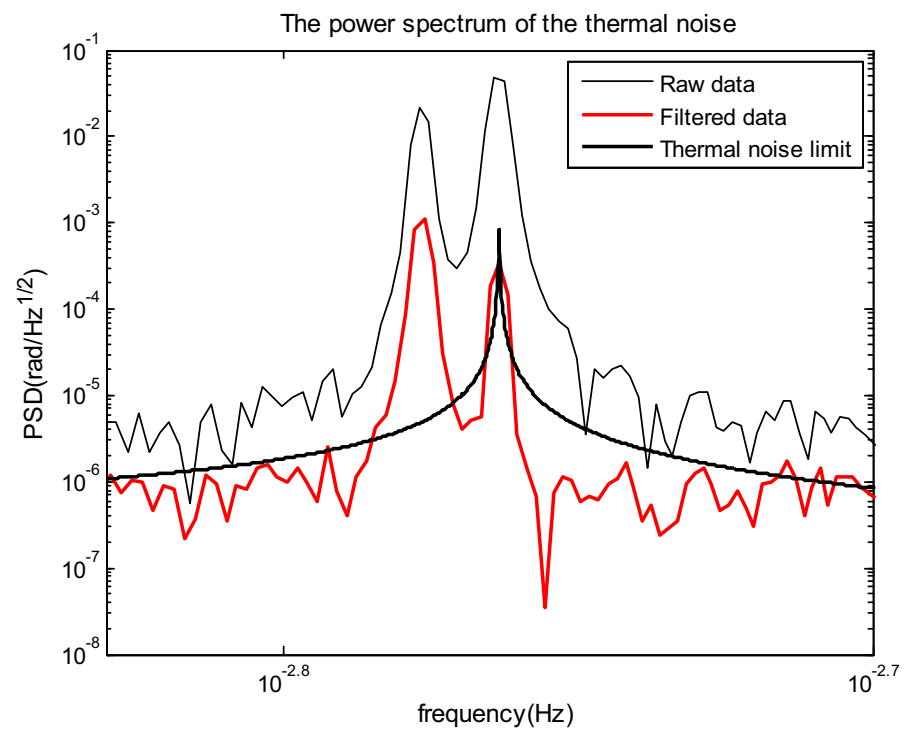

Fig. 6 (color online) The enlarged drawing of the black frame in Fig. 7 


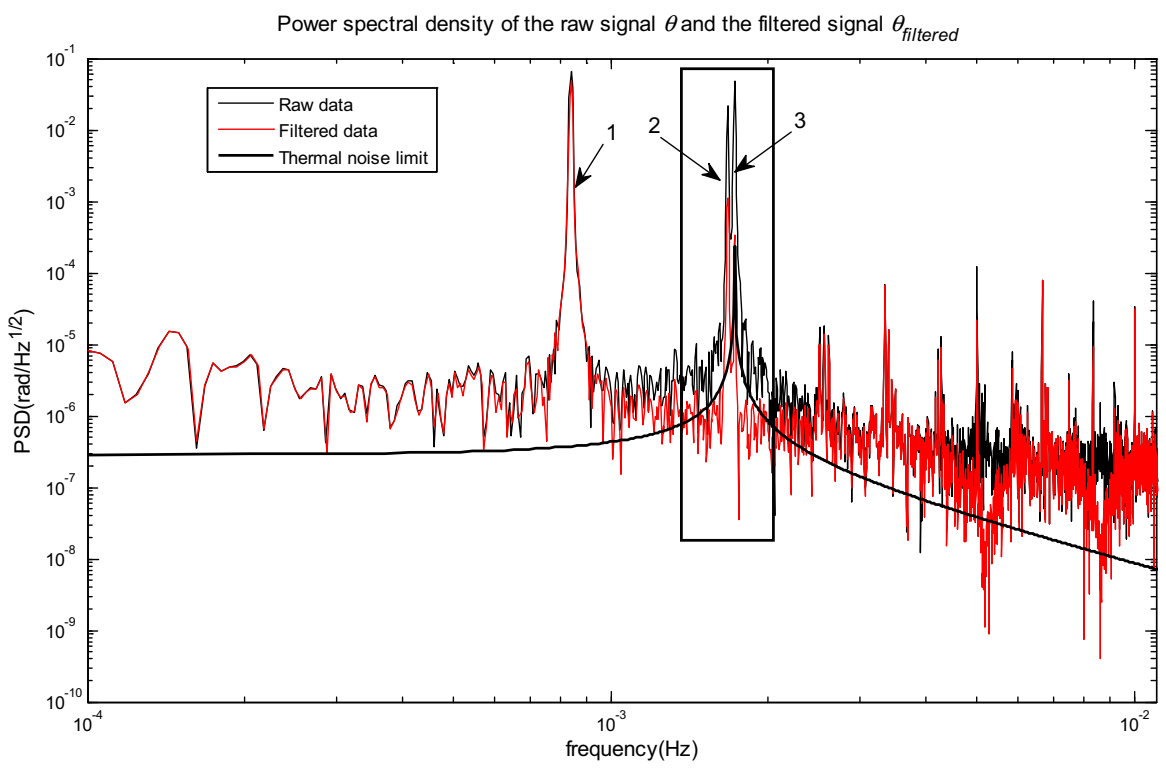

Fig. 7 (color online) Power spectral density of the raw signal $\theta$ and the filtered signal $\theta_{\text {filtered }}$. The blue, red and black lines denote the power spectral densities of the raw signal, filtered signal and thermal noise, respectively. The peaks 1,2 and 3 marks $1 \omega, 2 \omega$ and $\omega_{0}$ signal, respectively. The horizontal axis is the frequency, and the vertical axis is the amplitude. The details of the PSD between peak 2 and 3 are encircled by a black rectangle frame which shown in Fig. 6

irregularities in the rotation rate (in the Eq. (15), let $n$ equals 2). We could use a very symmetrical pendulum and keep rotation rate steady to suppress the $2 \omega$ signal if we need the improved gradiometer to obtain a higher measurement precision. The $\omega_{0}$ signal is the free torsion oscillation signal caused by the physical characteristic of the torsion fiber.

As the Fig. 6 show, we could use the frequency estimation method (Quinn 1994) to extract the period of the free torsional oscillation signal $T_{0}$. And then, we estimate the $W_{x z}$ and $W_{y z}$ by the following 5 steps of the data processing.

Firstly, we use a torsional filter ( $\mathrm{Su} \mathrm{1992)}$ to process the raw data in order to eliminate the $\omega_{0}$ signal. The torsional filter is to add two data points separated by $\pi$ of torsional phase, expressed as

$$
\theta_{\text {filtered }}(t)=\frac{1}{2}\left[\theta\left(t-\frac{T_{0}}{4}\right)+\theta\left(t+\frac{T_{0}}{4}\right)\right],
$$

where $T_{0}$ is the period of the free torsional oscillation signal and equals $579.9 \mathrm{~s}$ in our experiment. Then, the filtered signal $\theta_{\text {filtered }}(t)$ equals $\left\{\theta_{\text {filtered }}\left(t_{i}\right)\right\}$. In this way, the torsional oscillation signal will be significantly minimized in raw data. Meanwhile, the useful amplitude of the $1 \omega$ signal has also be attenuated by a factor $R(\omega)=\cos \left(\omega T_{0} / 4\right)(\mathrm{Su}$ 1992 ), and in our experiment, the $R(\omega)$ of the $1 \omega$ signal is about 0.72 . This attenuation needs to be corrected for the $1 \omega$ signal in result. The power spectral density of the amplitude of the filter data shows in Fig. 7. We find that the $\omega_{0}$ signal is decreased by about two orders, and the filtered $2 \omega$ signal is reduced to about the $1 / 25$ of the raw $2 \omega$ signal at the same time. The amplitude attenuation of the useful $1 \omega$ signal can be corrected to real amplitude in result. The data, which is usually two day long, has a irregular drift in 
temperature. Although this drift is small, its effect can be reduced by cutting the entire data into smaller segments and fitting each separately.

Secondly, each segment contains 3 periods of turntable, and then the filtered data is divided into 43 segments in the experiment. For the $j$ th segment, the filtered data is $\left.\left\{\theta_{\text {filtered }}^{j}\left(t_{i}\right)\right\}\right|_{j=1,2, \ldots, 43} ^{i=1,2, \cdots, 3 T}$, then the cut angular deflection signal $\theta_{c}^{j}(t)$ in the $j$ th segment could be express as

$$
\theta_{\text {cut }}^{j}(t)=\left.\left\{\theta_{\text {filtered }}^{j}\left(t_{i}\right)\right\}\right|_{j=1,2, \ldots, 43} ^{i=1,2, \ldots, 3600}
$$

Because each segment consists of an integer number of cycles, the harmonic terms in fit function are orthogonal to each other. The reason that we do not use shorter cuts is to ensure the harmonic terms approximately orthogonal to the drift terms and to have enough data points for a reliable fit (Choi 2006).

Thirdly, for every segment, we use the nonlinear least-squaring fitting method to fit the filtered signal points to extract the sine and cosine amplitude components of the $m \omega$ signal in the $j$ th segment. A similar nonlinear fitting method has been used in testing the equivalence principle by Gundlach et al. (1997). Base on the signal component of the experimental data, the goal fitted function could be written:

$$
\hat{\theta}_{c}^{j}(t)=b_{j}+\gamma_{j} t+\sum_{m=1}^{h}\left(a_{m, j}^{\cos } \cos (m \omega t)+a_{m, j}^{\sin } \sin (m \omega t)\right),
$$

where $b_{j}$ is constant term, $\gamma_{j}$ is liner drift coefficient, $m$ denotes the $m$ order harmonic of the useful signal and the highest item $h$ is set 9 , and $\left(a_{m, j}^{\mathrm{sin}}, a_{m, j}^{\mathrm{cos}}\right)$ is the (sine, cosine) amplitude
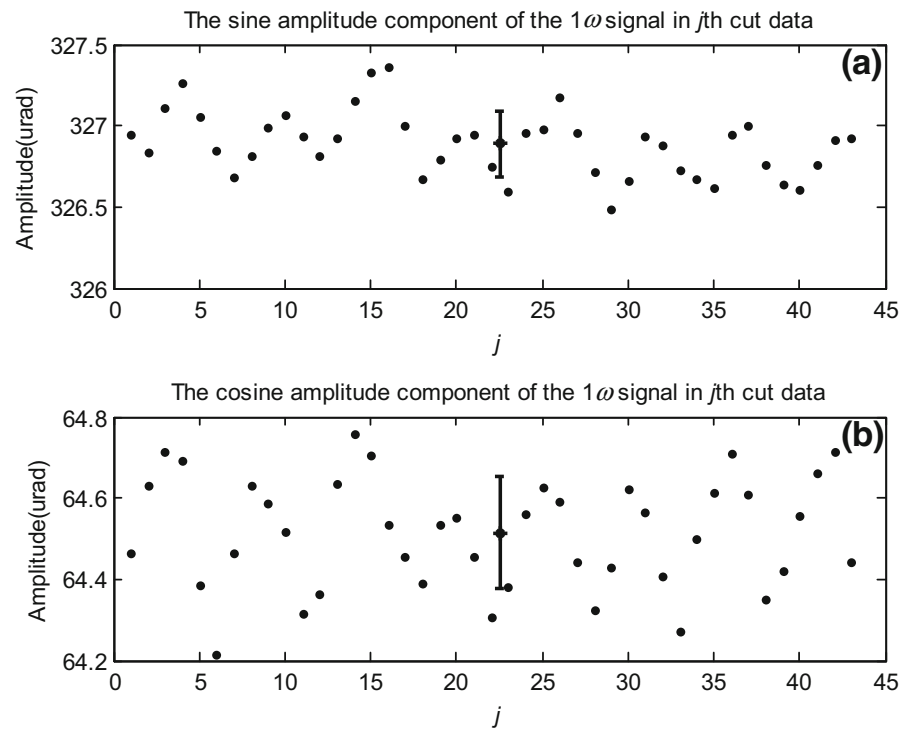

Fig. 8 The blank points are fitting value of the $1 \omega$ signal component coefficient in every segment. The asterisk and its error bar denote the final value and uncertainty of the corrected amplitude, respectively. a The $\tilde{a}_{1, j}^{\mathrm{sin}}$ is the sine amplitude component of the $1 \omega$ signal in $j$ th cut data. b The $\tilde{a}_{1, j}^{\cos }$ is the cosine amplitude component of the $1 \omega$ signal in $j$ th cut data 
components of the $m \omega$ signal in the $j$ th segment $(j=1,2, \ldots, 43)$, respectively. We use this function because the filtered data has not significant drift or has only linear drift which is removed by the torsional filter.

Later, we use the Chi Square to test our fitting result:

$$
\chi_{j}^{2}=\frac{1}{\sigma_{i}^{2}} \sum_{i=1}^{3600}\left[\left(\theta_{\text {cut }}^{j}\left(t_{i}\right)-\hat{\theta}_{\text {cut }}^{j}\left(t_{i}\right)\right]^{2},\right.
$$

where $\theta_{\text {cut }}^{j}\left(t_{i}\right)$ is the $j$ th segment of the filtered data, $\hat{\theta}_{\text {cut }}^{j}\left(t_{i}\right)$ is our fitting result for $j$ th segment filtered data. The $\sigma_{i}^{2}$ is every point standard deviation in the $j$ th segment filtered data and we let $\sigma_{i}$ equals $0.5 \mu \mathrm{rad}$ in our data process, the freedoms of the $j$ th segment filtered data $v$ equals 3580 . If $\chi_{j}^{2}>1$, then we consider that the $\hat{\theta}_{\text {cut }}^{j}\left(t_{i}\right)$ is not fit $\theta_{\text {cut }}^{j}\left(t_{i}\right)$ and it should be eliminated. In our experimental data that all 43 segments have been fitted eligible.

For the $j$ th segment, the estimated value of the amplitude components, $\left(a_{1, j}^{\mathrm{sin}}, a_{1, j}^{\cos }\right)$ are given by the above fitting accurately. These fitted amplitude components, $\left\{\left(a_{1, j}^{\mathrm{sin}}, a_{1, j}^{\cos }\right)\right.$, $j=1,2, \ldots, 43\}$ should be corrected to the real amplitude components, $\left\{\left(\tilde{a}_{1, j}^{\mathrm{sin}}, \tilde{a}_{1, j}^{\mathrm{cos}}\right), j=1\right.$, $2, \ldots, 43\}$ base on Eq. (17).

$$
\tilde{a}_{1, j}^{\sin }=a_{1, j}^{\sin } / R(\omega), \quad \tilde{a}_{1, j}^{\cos }=a_{1, j}^{\cos } / R(\omega) .
$$

Then, the corrected amplitude of the components is plotted in Fig. 8.

The average values, $\left(\bar{a}_{1}^{\text {sin }}, \bar{a}_{1}^{\text {cos }}\right)$ and error bars, $\left(\sigma \bar{a}_{1}^{\text {sin }}, \sigma \bar{a}_{1}^{\text {cos }}\right)$ of the $1 \omega$ signal component coefficient could be obtained by statistical and average for the $\left\{\left(\tilde{a}_{1, j}^{\mathrm{sin}}, \tilde{a}_{1, j}^{\mathrm{cos}}\right), j=1,2, \ldots\right.$, 43\}:

$$
\begin{aligned}
& \bar{a}_{1}^{\mathrm{sin}}=\frac{1}{43} \sum_{j=1}^{43} \tilde{a}_{1, j}^{\mathrm{sin}}, \quad \sigma \bar{a}_{1}^{\mathrm{sin}}=\sqrt{\frac{1}{43(43-1)} \sum_{j=1}^{43}\left(\tilde{a}_{1, j}^{\mathrm{sin}}-\bar{a}_{1}^{\mathrm{sin}}\right)^{2}}, \\
& \bar{a}_{1}^{\cos }=\frac{1}{43} \sum_{j=1}^{43} \tilde{a}_{1, j}^{\cos }, \quad \sigma \bar{a}_{1}^{\cos }=\sqrt{\frac{1}{43(43-1)} \sum_{j=1}^{43}\left(\tilde{a}_{1, j}^{\cos }-\bar{a}_{1}^{\mathrm{cos}}\right)^{2} .}
\end{aligned}
$$

Base on Eqs. (18) and (19), the result of the average value and the error bars could be written:

$$
\left\{\begin{array}{c}
a_{1}^{\sin }=(326.89 \pm 0.03) \mu \mathrm{rad} \\
a_{1}^{\cos }=(64.52 \pm 0.02) \mu \mathrm{rad}
\end{array}\right.
$$

The measurement results of the each segment are coincide within the error bar. According to Eq. (8), the measurement result of the $W_{x z}$ and $W_{y z}$ could be shown:

$$
\left\{\begin{array}{c}
W_{x z}=(-438.68 \pm 0.04) \mathrm{E} \\
W_{y z}=(86.58 \pm 0.03) \mathrm{E}
\end{array} .\right.
$$

For the whole experiment, the measurement accuracy $\sigma W_{x z}$ and $\sigma W_{y z}$ of the $W_{x z}$ and $W_{y z}$ are $0.04 \mathrm{E}$ and $0.03 \mathrm{E}$ respectively. We usually use a period of the turntable to complete a single measurement, and then the single measurement accuracy $\sigma_{1} W_{x z}$ and $\sigma_{1} W_{y z}$ of $W_{x z}$ and $W_{y z}$ could be show in Eq. (20) 


$$
\left\{\begin{array}{l}
\sigma_{1} W_{x z}=\sqrt{3 \times 43} \sigma W_{x z} \\
\sigma_{1} W_{y z}=\sqrt{3 \times 43} \sigma W_{y z}
\end{array},\right.
$$

where $3 \times 43$ is the number of the periods for the whole experiment.

The single measurement accuracy $\sigma_{1} W_{x z}$ and $\sigma_{1} W_{y z}$ of the improved gradiometer will extend to $0.45 \mathrm{E}$ and $0.32 \mathrm{E}$ respectively based on Eq. (20).

\section{Comparative measurement}

In order to the practical justification of the theoretical considerations, we develop an independent comparative measurement (Völgyesi and Ultmann 2012; Völgyesi 2015) before the formal measurement. As the Fig. 9 shows, we structure a calculable and knowable gravity gradient field $G G_{\mathrm{b}}$ by installing two lead blocks around the pendulum, and then test the responses (sensitivity) of the improved gradiometer to the artificial field $G G_{\mathrm{b}}$. According to Eq. (5), we could only consider the deflection angle $\theta(t)$ of the pendulum at the frequency $1 \omega$. In this way, we perform the comparative measurement by three steps. Step 1: we spend $15 \mathrm{~h}$ measuring the $\theta(t)$ of the torsion pendulum in the field $G G_{0}$ without setting the blocks, and the gravity gradient field is uniform and even. Step 2: we measure the $\theta(t)$ of the torsion pendulum in the field with setting the blocks about $9 \mathrm{~h}$, and this field is marked as $G G_{0}+G G_{\mathrm{b}}$. Step 3, we remove the lead blocks and remeasure $\theta(t)$ in the gravity gradient field $G G_{0}$.

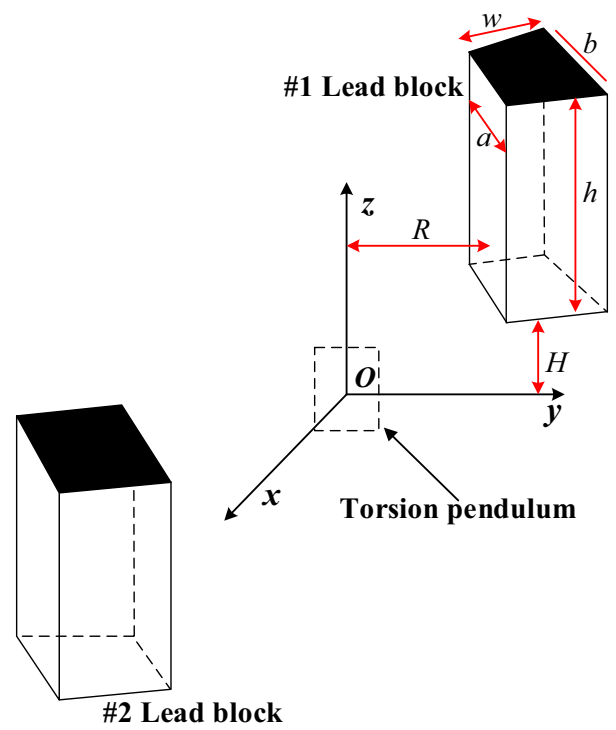

Fig. 9 (color online) Schematic drawing of the lead blocks and the pendulum. The \#1 and \#2 lead block are essentially the same blocks. The two blocks are symmetric about the origin of the lab frame $(O-x y z)$. The cross-section of the block is isosceles trapezoid. The upper and lower side length $a, b$ and the height of the trapezoid $w$ are equal to $9.39(5), 11.89(5)$ and $7.9(1) \mathrm{cm}$, respectively. The height of the block equals $26.0(1) \mathrm{cm}$. The bottoms of the \#1 and \#2 block are parallel with the plane $O-x y$. The distance $H$ between the bottom of the \#1 block with $O$-xy equals $18.3(5) \mathrm{cm}$, and the distance $R$ from the inside plane of the \#1 block to $O-x z$ equals $29.6(1) \mathrm{cm}$. The torsion pendulum is set in the dashed rectangle 

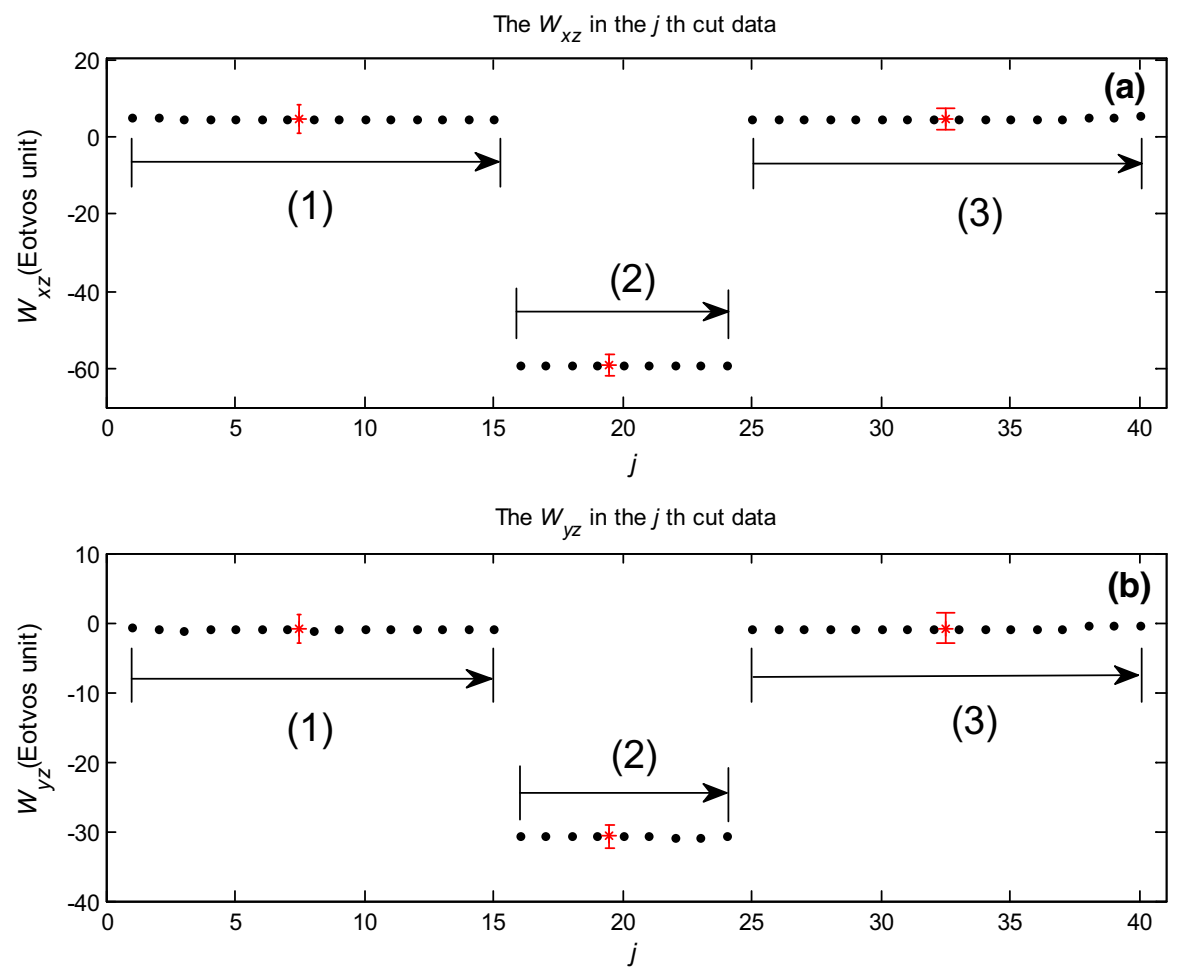

Fig. 10 (color online) The blank points are the fitting values of the $W_{\mathrm{xz}}$ and $W_{\mathrm{yz}}$ in every segment. The red asterisk and its error bar denote the final value and uncertainty of the corrected value, respectively. The number (1), (2) and (3) are labeled as the fields $G G_{0}, G G_{0}+G G_{\mathrm{b}}$ and $G G_{0}$, separately. There are 15,9 and 16 sets of $\left(W_{\mathrm{xz}}, W_{\mathrm{yz}}\right)$ in the fields $G G_{0}, G G_{0}+G G_{\mathrm{lb}}$ and $G G_{0}$, respectively. a: The $W_{\mathrm{xz}}$ in the $j$ th cut data. b: The $W_{\mathrm{yz}}$ in the $j$ th cut data

Table 1 The result of the independent comparative measurement and its corresponding theoretical value

\begin{tabular}{llllll}
\hline & Gravity gradient field & $W_{\mathrm{xz}}$ (theoretical) & $W_{\mathrm{yz}}$ (theoretical) & $W_{\mathrm{xz}}($ measured) & $W_{\mathrm{yz}}($ measured) \\
\hline Step 1 & $G G_{0}$ & - & - & $-4.61(6)$ & $-0.84(5)$ \\
Step 2 & $G G_{0}+G G_{\mathrm{b}}$ & - & - & $59.12(4)$ & $-30.73(1)$ \\
Step 3 & $G G_{0}$ & - & - & $-4.56(4)$ & $-0.91(3)$ \\
& $G G_{\mathrm{b}}$ & $63.69(5)$ & $-29.83(4)$ & $63.70(8)$ & $-29.85(6)$ \\
\hline
\end{tabular}

We measure the $\theta(t)$ of the torsion pendulum in the fields $G G_{0}, G G_{0}+G G_{\mathrm{b}}$ and $G G_{0}$ orderly. As the Fig. 10 shows, we obtained $W_{x z}$ and $W_{y z}$ of the field $G G_{0}$ with the same method we adopt in formal measurement. Then we use the spherical function expansion (Su 1992; Landau 2013) to calculate out the standardized 21th order multipole field $Q_{21}^{b}=[156(1)-i 73(3)] \times 10^{-9} / \mathrm{s}^{2}$ of the $G G_{\mathrm{b}}$, and further the theoretical $W_{x z}^{b}$ and $W_{y z}^{b}$ duo to the field $G G_{\mathrm{b}}$. And the relation between the $Q_{21}^{b}$ and the $W_{x z}^{b}, W_{y z}^{b}$ is (Landau 2013): 


$$
Q_{21}^{b}=\frac{1}{\sqrt{6}}\left(W_{x z}^{b}+i W_{y z}^{b}\right)
$$

With the same way we use in formal measurement, the result of the independent comparative measurement could be summarized in Table 1.

As the Table 1 shows, on the one hand the single measurement accuracies of the Steps 1-3 and the formal measurement are in good agreement. On the other hand, the measurement results of the Steps 1 and 3 are still in good agreement. That is to say, the measurement accuracy of the improved gradiometer is consistent. We can calculate the measured $W_{\mathrm{xz}}$ and $W_{\mathrm{yz}}$ of the field $G G_{\mathrm{b}}$ from the results of the Steps 1-3, and the measured $W_{\mathrm{xz}}$ and $W_{\mathrm{yz}}$ are agree with the theoretical value. It is mean that the gradiometer could response the artificial field $G G_{\mathrm{b}}$ correctly. Therefore, the independent comparative measurement indicate that the measurement of the improved gradiometer is stable and effective.

\section{Conclusions}

In the measurement of the gravity gradients with the traditional torque type gradiometer, it is of inefficiency and with relatively low accuracy due to the static operating mode. To improve the efficiency and accuracy, we develop an improved torque type gradiometer by using dynamic modulation mode. In the dynamic modulation mode, the torsion pendulum is mounted on a continuously rotating turntable with a constant rate, and the gravity gradients are extracted from the deflection angle signal that is modulated by the turntable. This improvement shortens the measurement cycle largely and improves the accuracy of the obtained gravity gradients effectively. The experimental results show that at the same accuracy of $1 \mathrm{E}$, the measurement cycle of the improved torque type gradiometer is only about one thirty-sixth of the traditional gradiometer. In the measurement of the gravity gradient by the improved torque type gradiometer with dynamic modulation, the uncertainties of the obtained gravity gradient components $W_{x z}$ and $W_{y z}$ are $0.45 \mathrm{E}$ and $0.32 \mathrm{E}$ respectively, which are more precise than those obtained by the traditional torque type gradiometer. The results of independent comparative measurement show that the measurement of the improved gradiometer is stable and effective. The errors of the thermal noise on the gravity gradient components $W_{x z}$ and $W_{y z}$ are $0.01 \mathrm{E}$. The errors of the irregularities in the rotation rate on the gravity gradient components $W_{x z}$ and $W_{y z}$ are 0.03 E. The improved torque type gradiometer with dynamic modulation is highly efficient and accurate. Because of the short measurement cycle, the observation can avoid many noises and disturbances from external environment, and hence this improved torque type gradiometer may have a wide potential in practical applications. Besides, due to the configuration of our instrument and the particular positions of the four test mass, the preliminary experiment of the gravity gradient with our improved gradiometer determines two components $W_{x z}$ and $W_{y z}$ of the gravity gradient. As long as we alter the positions of the four test mass, our improved gradiometer can also obtain the four components of the gravity gradient like the traditional gradiometer. It is instructive and significant to improve the torque type gradiometer measuring the gravity gradient. Since the gradiometer is a field device that must operate in the field condition and the carrying and working conditions in field are relative unsettled, we would adopt a relative stable but insensitive metallic leaf to replace the fiber. That is to say, we must find a balance between sensitivity and stability. If 
some more improvement can be performed, our improved gradiometer can be used to engineering application promisingly. Since the configuration of the instrument and the position of test mass are not fully understood, there are still many works needed performing in the future.

Acknowledgements This work is partially supported by the National Natural Science Foundation of China (Grant Nos. 11575160, 91636221, 11605065).

\section{References}

Barton DC (1931) Gravity measurements with the Eotvos torsion balance. Consulting Geologist and Geophysicist, Houston, Texas. Physics of the Earth 77:167-188

Bell RE, Hansen RO (1998) The rise and fall of early oil field technology: the torsion balance gradiometer. Lead Edge 17(1):81-83

Choi KY (2006) A new equivalence principle test using a rotating torsion balance. Ph.D

Dehlinger P (1978) Marine gravity, vol 22. Elsevier, Amsterdam

DiFrancesco D, Grierson A, Kaputa D, Meyer T (2009) Gravity gradiometer systems_advances and challenges. Geophys Prospect 57(4):615-623

ELCOMAT 3000, Germany Moeller-Wedel Optical GmbH. https://www.haag-streit.com/de/moeller-wedeloptical/

Goodfellow Cambridge Limited, Huntingdon PE29 6WR, England. http://www.goodfellow.com/contact-us/

Gundlach JH, Smith GL, Adelberger EG, Heckel BR, Swanson HE (1997) Short-range test of the equivalence principle. Phys Rev Lett 78(13):2523

Kirkup L, Frenkel RB (2006) An introduction to uncertainty in measurement: using the GUM (guide to the expression of uncertainty in measurement). Cambridge University Press, Cambridge

Lancaster-Jones E (1932) The principles and practice of the gravity gradiometer. J Sci Instrum 9(11):341

Landau LD (ed.) (2013) The classical theory of fields, vol 2. Elsevier, Amsterdam

Luo J, Shao CG, Tian Y, Wang DH (2013) Thermal noise limit in measuring the gravitational constant G using the angular acceleration method and the dynamic deflection method. Phys Lett A 377(21):1397-1401

Quinn BG (1994) Estimating frequency by interpolation using Fourier coefficients. IEEE Trans Signal Process 42(5):1264-1268

Rankine AO (1932) On the representation and calculation of the results of gravity surveys with torsion balances. Proc Phys Soc 44(4):465

Saulson PR (1990) Thermal noise in mechanical experiments. Phys Rev D 42(8):2437

Schweydar W (1918) Die Bedeutung der Drehwaage von Eötvös für die geologische Forschung nebst Mitteilung der Ergebnisse einiger Messungen. Zeitschrift für praktische Geologie 26:157-162

Shaw H, Lancaster-Jones E (1922) The Eötvös torsion balance. Proc Phys Soc Lond 35(1):151

SP-400, Shanghai Shanjin Vacuum Equipment Co., Ltd., Shanghai, China

Su Y (1992) A new test of the weak equivalence principle. Ph.D

Szabó Z (2016) The history of the 125 year old Eötvös torsion balance. Acta Geod Geoph 51(2):273-293

Tu LC, Li Q, Wang QL, Shao CG, Yang SQ, Liu LX, Liu Q, Luo J (2010) New determination of the gravitational constant G with time-of-swing method. Phys Rev D 82(2):022001

Völgyesi L (2001) Local geoid determination based on gravity gradients. Acta Geodaetica et Geophysica Hungarica 36(2):153-162

Völgyesi L (2015) Renaissance of torsion balance measurements in Hungary. Periodica Polytechnica Civ Eng 59(4):459

Völgyesi L, Ultmann Z (2012) Reconstruction of a torsion balance and the results of the test measurements. In: Kenyon SC, Pacino MC, Marti UJ (eds) Geodesy for planet earth. Springer, Berlin, pp 281-289

Zhou MK, Duan XC, Chen LL, Luo Q, Xu YY, Hu ZK (2015) Micro-Gal level gravity measurements with cold atom interferometry. Chin Phys B 24(5):050401 